\title{
Association of total serum cholesterol with functional outcome following home care rehabilitation in Italian patients with stroke
}

\author{
Maria Concetta Cataldo, M.D. ${ }^{a}$, Maria Luisa Calcara, M.D. ${ }^{a}$, Giuseppe Caputo, M.D. ${ }^{a}$, \\ and Caterina Mammina, M.D.,* \\ ${ }^{\mathrm{a}}$ Geriatric Assessment and Integrated Home Care Unit, District 10, Regional Health Agency 6, Palermo, Italy \\ ${ }^{\mathrm{b}}$ Department of Sciences for Health Promotion "G. D'Alessandro", University, Palermo, Italy
}

\begin{abstract}
Background: Stroke is a disabling disease. In elderly populations, stroke is the third leading cause of death and the primary cause of reduction in or loss of functional ability and personal autonomy. Possible associations between levels of total serum cholesterol (TC) and both incidence of stroke and functional outcomes after rehabilitation are still under study.

Objective: To detect positive and negative prognostic factors associated with functional outcomes in first-time stroke patients admitted to an integrated home care rehabilitative program.

Methods: This study enrolled 141 patients with a first-time stroke who were admitted to a home care rehabilitation program. Primary outcome measures were the Barthel activities of daily living (ADL) and mobility indices at the beginning and end of the rehabilitative treatment. The impact of TC and other demographic and clinical variables was analyzed using bivariate and multivariate logistic regression analyses.

Results: Age and Short Portable Mental Status Questionnaire (SPMSQ) score were negatively associated with functional outcome. In contrast, elevated TC was positively associated with a better home rehabilitative treatment outcome. Barthel index score at admission was negatively associated with outcomes assessed by the Barthel ADL index and age with outcomes assessed by the Barthel mobility index. In a multivariate logistic regression analysis, SPMSQ score and elevated TC were significantly associated with outcome. Specifically, higher SPMSQ scores were negatively associated with better rehabilitative treatment outcomes, whereas elevated TC was positively associated.

Conclusions: Elevated TC seems to be associated with better functional outcomes in patients with first-time stroke. (C) 2012 Elsevier Inc. All rights reserved.
\end{abstract}

Keywords: Total serum cholesterol; Barthel indices; Stroke; Home care; Rehabilitation

It is widely known that stroke is a disabling disease. In elderly populations, stroke is the third leading cause of death and the primary cause of reduction in or loss of functional ability and personal autonomy [1]. The huge social burden of this disease highlights the importance of identifying prognostic factors as early as possible, because despite therapeutic advances, only a small percentage of elderly stroke survivors achieve a full recovery and most show varying degrees of reduced functional autonomy [2]. This greatly affects their quality of life, often making it unsatisfactory and jeopardizing previously satisfactory household relationship dynamics [3].

According to data from the 2001 Italian National Institute of Statistics, approximately 196,000 new strokes occur

\footnotetext{
The authors report no conflicts of interest.

* Corresponding author: Department of Sciences for Health Promotion “G. D’Alessandro", University Via del Vespro 133, I-90127 Palermo, Italy.

E-mail address: caterina.mammina@unipa.it (C. Mammina).
}

every year in Italy, with crude incidence rates ranging between 1.5 and 2.8 per 1000 inhabitants. In addition, a marked upward south to north trend is observed [4,5]. Incidence increases with age and peaks in the over 85 population $[4,5]$. The acute poststroke mortality rate is $23 \%$, and the mortality rate within 1 year is approximately $30 \%$ [4,5]. It is estimated that $12 \%-15 \%$ of stroke survivors are hospitalized in rehabilitation units, one third have a severe disability and a marked limitation in activities of daily living, 20\% need assistance to walk, and $70 \%$ are unable to return to their previous job [4,5].

Assessing factors that correlate with functional outcome is an important issue in stroke and disability research. Previous studies have shown a consistent relationship between coronary heart disease and high total serum cholesterol (TC) levels. The correlation between stroke and TC remains unclear, and currently very little data are available regarding the effect of cholesterol on survival in patients after stroke [6,7]. Until today, few studies have 
examined the possible association between plasma levels of $\mathrm{TC}$ and the incidence of stroke, and the epidemiologic data are conflicting. However, the association between the treatment of high TC and recurrence of stroke or mortality has been actively investigated [8-10]. The Prospective Evaluation of Pravastatin in the Elderly (PROSPER) study, which investigated the impact of pravastatin treatment in 5804 elderly subjects with either a history of vascular disease or a high-risk profile, found that after a mean follow-up of 3.2 years, pravastatin treatment significantly reduced the relative risk for fatal or nonfatal stroke by $15 \%$ [8]. More recently, the Stroke Prevention by Aggressive Reduction in Cholesterol Levels (SPARCL) trial, recruiting 4731 patients with a history of recent stroke or transient ischemic attack (TIA), found that atorvastatin reduced the overall incidence of stroke, despite a small increase in the incidence of hemorrhagic stroke [9]. Finally, in a recent systematic review examining interventions in the management of serum lipids for preventing stroke recurrence, statin therapy in patients with a history of ischemic stroke or TIA was associated with a significant reduction in subsequent major coronary events, but only a marginal reduction in the risk of stroke recurrence (odds ratio [OR] 0.88, 95\% confidence interval [CI] 0.77-1.00) [8]. Accordingly, the authors conclude that patients with ischemic stroke or TIA should receive statins [10].

In Italy, in accordance with the national Guidelines on Rehabilitation Activities [11,12], patients with strokerelated disability are included, after their hospital discharge, in an integrated social and health care pathway aimed at eliminating or minimizing disability so that the patient can reach the highest level of independence possible in both their social and working life [11,12]. The primary tool in this pathway is a customized rehabilitation program. This program contains a specific plan created by a rehabilitation team that is coordinated by a physiatrist. Such a customized rehabilitation program considers residual and recoverable abilities, the needs and preferences of the patients and their families, and possible obstacles to program adherence. The program also defines the role of the rehabilitation team, taking into consideration all actions needed to achieve the planned outcome. Necessary actions are determined by defining short-, medium-, and long-term objectives and the expected timeline for reaching them. The objectives are then periodically updated by the team according to recovery progress. The rehabilitation program identifies the healthcare workers involved in each intervention and their specific roles $[11,12]$.

The Geriatric Assessment and Integrated Home Care (GAIHC) Unit of District 10 of the Regional Health Agency (ASP6) of Palermo carried out a prospective cohort study on elderly patients over 65 years having a first-time stroke, who were admitted to home care after hospital discharge. All patients were treated at home with a customized rehabilitation program as summarized above [11-12].

\section{Hypothesis}

This study was conducted to detect associations of demographic and clinical factors with functional outcomes of patients treated at home after a stroke. In particular, the role of TC as a prognostic factor was investigated.

\section{Methods}

This was a prospective cohort study that enrolled 141 patients having sustained a first-time stroke between December 2007 and December 2009 in an inner-city area of Palermo, Italy. All patients who were admitted to the rehabilitative program of the GAIHC Unit at the Health District 10, Local Health Authority 6 of Palermo, Italy, were included, regardless of age, stroke severity, or comorbid conditions. The Unit is comprised of an internal medicine specialist, a physiatrist, a social assistant, a registered nurse, a physiotherapist, and a home healthcare nurse, in collaboration with a geriatrician and a neuropsychiatrist at the local district health clinic.

On both admission and discharge, all patients included in the study were given a standardized evaluation based on a multidimensional assessment including medical, sociodemographic, and environmental components, as well as functional components related to activities of daily living (ADLs) and mobility assessed with the Barthel scales [13-17]. A comorbidity index was calculated using the Cumulative Illness Rating scale (CIRS), and the Short Portable Mental Status Questionnaire (SPMSQ) was administered. A rehabilitation program was formulated based on the collected data, i.e., information from the standardized clinical measures, laboratory tests results, and clinical impressions gathered from all components of the GAIHC. Information was obtained from relatives or caregivers when necessary.

Stroke was defined according to criteria set forth by the World Health Organization [18]. Patients with TIAs or subarachnoid hemorrhages were not included. Stroke type (hemorrhage/infarct) and size and site of lesion were determined using computerized tomography (CT) scans.

The outcome of the rehabilitative treatment was quantified as percent change scores for both Barthel ADL and mobility indices. These were found by calculating the ratio of the mean difference between scores at the beginning and end of the treatment period to the score at the beginning.

The following predictors of rehabilitative treatment outcome were investigated: age, sex, stroke subtype according to the Oxfordshire Community Stroke Project (OCSP) classifications [19], diabetes mellitus, ischemic heart disease (IHD), hypertension, and TC. Diabetes was considered present when a patient had known diabetes mellitus on admission or when their fasting plasma glucose level was $>126$ $\mathrm{mg} / \mathrm{dL}$ on admission. IHD was present when a patient had a history of IHD or when IHD had been diagnosed during the hospital stay. Hypertension was classified as present when a patient had been diagnosed during the hospital stay, was receiving antihypertensive treatment before admission, or 
Table 1

Demographic and Clinical Characteristics of the Patients $(n=141)$

\begin{tabular}{lc}
\hline Variable & Value $^{\mathrm{a}}$ \\
\hline Female gender & $85(60.3)$ \\
Age & $77.33 \pm 9.02$ \\
Hypercholesterolemia & $32(22.7)$ \\
Diabetes mellitus & $51(36.2)$ \\
Ischemic heart disease & $68(48.9)$ \\
Atrial fibrillation & $11(7.8)$ \\
Hypertension & $117(83.0)$ \\
CIRS comorbidity index & $2.12 \pm 0.47$ \\
SPQMQ score & $5.38 \pm 3.71$ \\
Ischemic stroke & $116(82.3)$ \\
Stroke subtype (OCSP) & \\
LACI & $53(45.7)$ \\
PACI & $37(31.9)$ \\
POCI & $9(7.8)$ \\
TACI & $17(14.7)$ \\
Admit Barthel ADL Index score & $50.86 \pm 10.30$ \\
Admit Barthel mobility Index score & $36.27 \pm 5.82$ \\
\hline
\end{tabular}

LACI = Lacunar infarction; PACI = Partial anterior circulation infarction; $\mathrm{POCI}=$ Posterior circulation infarction; $\mathrm{TACI}=$ Total anterior circulation infarction; $\mathrm{ADL}=$ Activities of Daily Living.

${ }^{\mathrm{a}}$ Values are mean $\pm \mathrm{SD}$ or $\mathrm{n}(\%)$ unless otherwise stated.

when hypertension was diagnosed on admission by repeated detection of blood pressure $\geqslant 160 / 95 \mathrm{~mm} \mathrm{Hg}$. TC was determined in a blood sample drawn on admission to the homecare rehabilitative treatment program, and a cutoff level of $200 \mathrm{mg} / \mathrm{dL}$ was used to classify patients as having high serum TC levels. Statin use was not included as a prognostic factor because statins were prescribed to only 3 patients.

\section{Statistical analysis}

Statistical analysis was performed using the software programs EpiInfo ver. 3.5 (CDC, Atlanta, Georgia) and Statistica (StatSoft, Inc, Tulsa, Oklahoma). Descriptive analyses were performed by calculating means with standard deviations (SD), and frequencies, and then assessing differences using 1-way analysis of variance (ANOVA) or KruskalWallis when appropriate, or a chi-square test or Fisher exact test, respectively. Associations between the variables under examination were evaluated using contingency tables. The crude effect of each variable on treatment outcome was initially analyzed; then multivariate logistic regression analysis was used to estimate the adjusted effects of the prognostic factors, after dichotomizing the Barthel percent changes into positive versus negative or zero values. The statistical criterion for eliminating a variable from the model was a significance level of 0.10 . Age and sex were considered basic elements and retained in the model. Results of statistical tests were considered significant for $p$ values $<0.05$.

\section{Results}

\section{Demographic and clinical characteristics of patients}

Table 1 shows the demographic and clinical characteristics of the participants, presented as mean $\pm \mathrm{SD}$. The mean age at the time of stroke was $77.33 \pm 9.02$ years, and the sample was $60.3 \%$ female. The mean age for women was $79.46 \pm 8.52$ years, and for men, $74.09 \pm 8.85$ years $(p<0.001)$.

Patients with ischemic stroke did not significantly differ from those with hemorrhagic stroke in age distribution (ischemic versus hemorrhagic, $77.94 \pm 8.20$ versus $74.48 \pm$ 11.92 years, $p=0.08)$, comorbidity index $(2.15 \pm 0.40$ versus $2.02 \pm 0.69, p=0.21)$, SPMSQ value $(5.36 \pm$ 3.65 versus $5.44 \pm 4.03, p=0.09$ ), frequency of hypertension $(81.9 \%$ versus $88.0 \%, p=0.34)$, Barthel ADL or mobility scores on admission (ADL: $50.65 \pm 10.26$ versus $51.88 \pm 10.63, p=0.59$ ), (mobility: $36.02 \pm 5.90$ versus $37.44 \pm 5.35, p=0.27$ ), or length of rehabilitative treatment $(70.31 \pm 35.65$ versus $66.92 \pm 33.12$ days, $p=$ 0.66 ). They differed slightly by gender (ischemic versus hemorrhagic, percent female $63.8 \%$ versus $44.0 \%, p=$ $0.05)$ and frequency of diabetes $(39.7 \%$ versus $20.0 \%$, $p=0.05)$.

Thirty-two subjects had been diagnosed with high TC, but only 3 took medication for it. TC did not appear to be significantly associated with ischemic stroke: high TC was present in 29 of $116(25.0 \%)$ patients having sustained an ischemic stroke and 3 of $25(12.0 \%)$ with hemorrhagic stroke $(p=0.12)$. Considering the results obtained and the relatively small proportion of hemorrhagic strokes, stratification by stroke subtype was not plausible and the subsequent analysis was performed on all patients regardless of stroke pathogenesis.

\section{Follow-up and prognostic factors}

The mean length of the rehabilitative treatment period was $69.71 \pm 35.12$ days. The outcome of the rehabilitative treatment was assessed by comparing the Barthel ADL and mobility scores at the beginning and the end of the period. The associations of outcome with the demographic and clinic predictors under investigation, obtained using bivariate analysis, are summarized in Tables 2 and 3.

High TC was positively and significantly associated with better home rehabilitative treatment outcome (Table 2). Presence of IHD was negatively associated with treatment outcome (Table 2). Age and SPMSQ score were also significantly associated with outcome using both Barthel ADL and mobility scores. Barthel indices at admission appeared to be negatively associated with outcome, because of a ceiling effect (Table 3). Bivariate analysis failed to show a statistically significant association between the comorbidity index and rehabilitation program outcome (Table 3). Similarly, stroke subtype was not associated with outcome in either ADLs or mobility (Table 2).

Demographic and clinical characteristics were not significantly different between the high TC patients and those with normal TC levels (Table 4).

When the significant variables, i.e., high TC, presence of IHD, SPMSQ score, and Barthel indices at admission, 
Table 2

Outcome of the Rehabilitative Treatment Measured as Percent of Difference on Barthel Index Scores in Relation with the Categorical Variables Investigated as Outcome Predictors. Results of the Bivariate Analysis ( $\mathrm{n}=141$ patients)

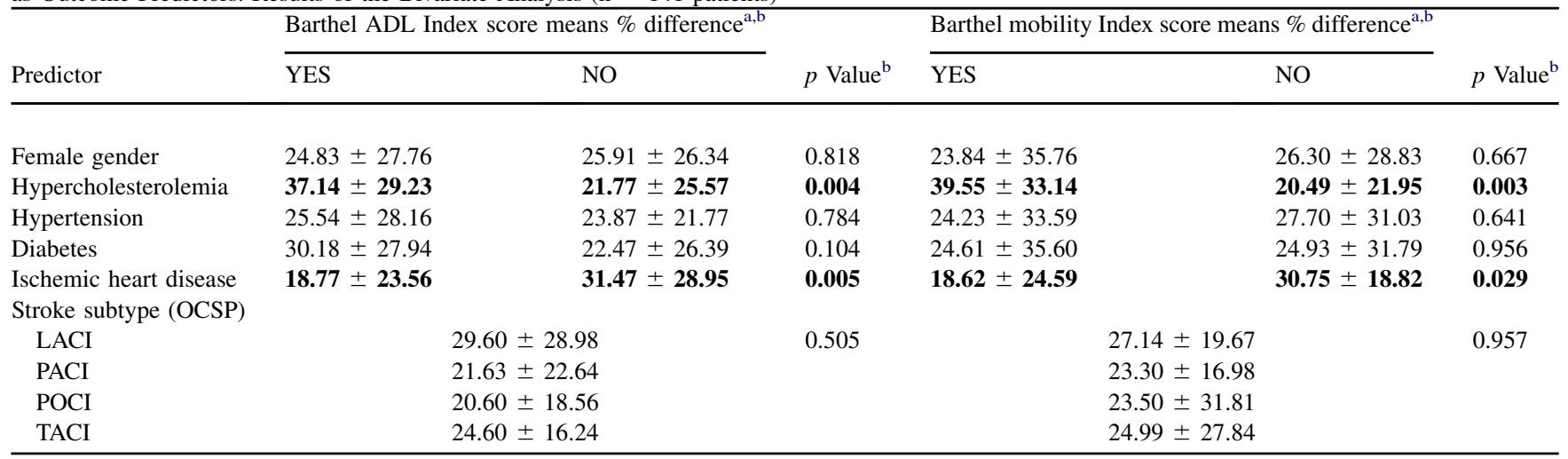

$\mathrm{ADL}=$ Activities of Daily Living; LACI $=$ Lacunar infarction; PACI $=$ Partial anterior circulation infarction; POCI $=$ Posterior circulation infarction; $\mathrm{TACI}=$ Total anterior circulation infarction.

${ }^{\mathrm{a}}$ Values are mean $\pm \mathrm{SD}$.

${ }^{\mathrm{b}}$ In bold statistically significant values.

along with age and gender, were assessed in a multivariate logistic regression analysis, only SPMSQ score and high TC were consistently associated with better scores on both Barthel indices (Table 5).

\section{Discussion}

The current study adds to the mounting evidence that patients with high TC levels have better functional outcomes following rehabilitation. Indeed, corroborating the results of previous studies, in our patients only SPMSQ score and TC showed a significant association with functional outcome following the home rehabilitative program. SPMSQ score is largely known to be negatively associated with stroke outcomes, thus the positive association between rehabilitation outcome and higher levels of TC in both bivariate analysis and after adjusting for confounding factors is of particular interest.

Little is known about the prognostic role of cholesterol on functional outcome after a stroke. Several authors

Table 3

Outcome of the Rehabilitative Treatment Measured as Percentage Change Barthel Indices Scores in Relation with the Quantitative Variables Investigated as Outcome Predictors, After Stratifying According to the Quartile Values. Results of the Bivariate Analysis ( $\mathrm{n}=141$ patients)

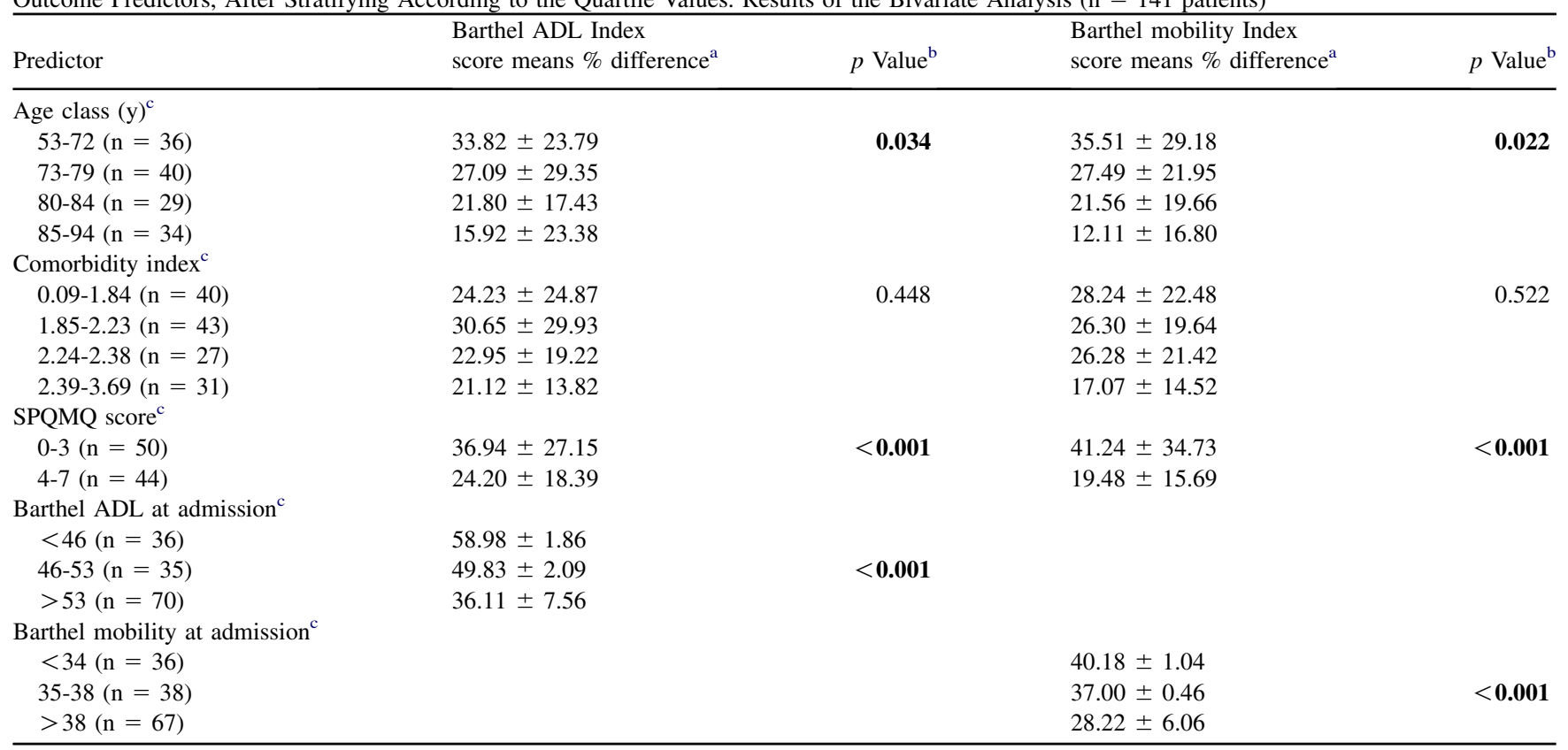

$\mathrm{ADL}=$ Activities of Daily Living; SPQMQ = Short Portable Mental Status Questionnaire.

${ }^{a}$ Values are mean $\pm \mathrm{SD}$.

${ }^{\mathrm{b}}$ In bold statistically significant values.

${ }^{c}$ In brackets number of patients by stratum. 
Table 4

Main Demographic and Clinical Characteristics of the Patients Under $\underline{\text { Study After Stratifying By Presence or Absence of High Serum TC }{ }^{\text {a Level }}}$

\begin{tabular}{llll}
\hline & \multicolumn{2}{l}{ High TC $^{\mathrm{b}}$} & \\
\cline { 2 - 3 } Variable & $\begin{array}{l}\text { Presence } \\
(\mathrm{n}=32)\end{array}$ & $\begin{array}{l}\text { Absence } \\
(\mathrm{n}=109)\end{array}$ & $p$ Value \\
\hline Female gender & $18(56.3)$ & $67(61.5)$ & 0.30 \\
Age & $75.12 \pm 7.30$ & $77.97 \pm 9.39$ & 0.12 \\
Diabetes mellitus & $11(34.4)$ & $40(36.7)$ & 0.41 \\
Ischemic heart disease & $14(43.8)$ & $55(50.5)$ & 0.26 \\
Hypertension & $89(87.5)$ & $28(81.7)$ & 0.23 \\
CIRS comorbidity index & $2.23 \pm 0.43$ & $2.09 \pm 0.47$ & 0.16 \\
SPQMQ score & $4.84 \pm 3.93$ & $5.54 \pm 3.64$ & 0.35 \\
Admit Barthel ADL & $48.31 \pm 11.54$ & $51.61 \pm 9.84$ & 0.11 \\
$\quad$ Index score & $35.59 \pm 5.37$ & $36.47 \pm 5.95$ & 0.46 \\
Admit Barthel mobility & & & \\
$\quad$ Index score & & & \\
Rehabilitation treatment days & $77.41 \pm 33.18$ & $67.45 \pm 35.50$ & 0.16 \\
\hline \multicolumn{2}{c}{ a TC $=$ serum total cholesterol. } & & \\
$\quad$ b Values are mean \pm SD or $\mathrm{n}(\%)$ unless otherwise stated. &
\end{tabular}

[20-25] have studied the influence of cholesterolemia on survival in patients with sequelae of stroke and all have found that higher TC levels were associated with a lower risk of death. Dyker et al. [20] first analyzed the influence of TC on survival after stroke and found that higher serum TC level was associated with lower 3-month overall mortality. However, data examining the relationship between TC and the dynamic process of functional recovery after a stroke are lacking. Previous studies [2025 ] have reported an inverse association between serum cholesterol levels and severity of stroke, suggesting that higher levels of serum cholesterol could be associated with so-called minor ischemic strokes. Vauthey [21] and Zuliani et al. [22] reported that patients with high TC levels had a lower risk of death or poor functional outcome in the first month after ischemic stroke. More recently, this association has been confirmed by the study of Pan et al. [25]. By using serial Barthel Index scores until 6 months after stroke as the

Table 5

Multivariable Logistic Regression Analysis of the Prognostic Factors, After Dichotomizing the Barthel Percentage Changes (Positive Versus Negative or Zero Values)

\begin{tabular}{|c|c|c|c|}
\hline & Variable $^{a}$ & AOR $(95 \% \mathrm{CI})^{\mathrm{b}}$ & $\overline{p \text { Value }}$ \\
\hline \multirow[t]{5}{*}{ Barthel ADL } & Age & $0.96(0.91-1.00)$ & 0.07 \\
\hline & Gender (female) & $0.69(0.29-1.66)$ & 0.41 \\
\hline & SPQMQ score & $0.85(0.74-0.97)$ & 0.02 \\
\hline & High TC level $^{c}$ & $3.37(1.10-10.25)$ & 0.03 \\
\hline & $\begin{array}{l}\text { Admit Barthel } \\
\text { ADL Index }\end{array}$ & $0.94(0.89-0.99)$ & 0.04 \\
\hline \multirow[t]{5}{*}{ Barthel mobility } & Age & $0.90(0.94-0.99)$ & 0.02 \\
\hline & Gender (female) & $0.53(0.22-1.24)$ & 0.14 \\
\hline & SPQMQ score & $0.83(0.74-0.94)$ & $<0.05$ \\
\hline & High TC level $^{\mathrm{c}}$ & $3.30(1.16-9.38)$ & 0.02 \\
\hline & $\begin{array}{l}\text { Admit Barthel } \\
\text { mobility Index }\end{array}$ & $0.94(0.87-1.02)$ & 0.17 \\
\hline
\end{tabular}

\footnotetext{
${ }^{\text {a }}$ In bold statistically significant values.

b $\mathrm{AOR}=$ adjusted odds ratio.

c $\mathrm{TC}=$ serum total cholesterol.
}

main outcome measures, Pan et al. demonstrated that the serum TC level at the acute stage of ischemic stroke is an independent predictor of long-term functional outcome. Furthermore, although the size of ischemic injury has undoubtedly proven to be predictive of functional outcome, even after adjusting for the effects of lesion size and baseline Barthel index scores, cholesterol was still a significant prognostic factor related to better rehabilitation outcome [20-27].

This implies that favorable functional results in the presence of high cholesterol levels cannot be fully explained by the association between high cholesterol and strokes of minor severity. As a matter of fact, at this moment there are no established biologic mechanisms that explain these results. Some studies have suggested a neuroprotective role of cholesterol that could be attributed to its antioxidant properties [28]. Other studies have suggested that low HDL cholesterol in the blood enhances the action of platelet aggregation by acting on the platelet activating factor $[29,30]$. In addition, high concentrations of cholesterol might have a neuroprotective effect by modulating the action of the gamma glutamyltransferase and acetylcholinesterase enzymes [31]. Alternately, other authors suggest that lower TC levels in elderly patients may be a proxy for poorer general health status and/or malnutrition, both conditions leading to adverse functional outcomes [32].

Another issue to consider is a possible favorable effect of the traditional Mediterranean diet that is likely adopted by elderly patients during their lifetime in Sicily. It is indeed well known that a Mediterranean diet can exert protective effects against stroke and cardiovascular disease via a number of its components, such as fruits and vegetables, whole grains, and fatty fish. All of these have been associated with a reduction in the risk for stroke and hypertension, the major modifiable risk factor for stroke $[33,34]$.

Finally, a concerning finding in this study was the number of patients with elevated TC levels who were not on statin therapy. Although a number of guidelines have been published supporting the effectiveness of statins in reducing the risk of cardiovascular events, high variability in the prescription of such drugs has been reported in elderly people $[35,36]$. Indeed, only one fourth of patients who are eligible for statin therapy received it, and numbers are particularly low among individuals $\geqslant 75$ years old $[35,36]$. The undertreatment of high TC levels in elderly persons can be attributed to a number of possible causes. First, in some studies the predictive value of cholesterol has been shown to decline with increasing age $[35,36]$. Second, statin treatment may be perceived as unlikely to benefit older patients because of their theoretical shortlife expectancy [35,36]. Finally, physicians may have misconceptions about the risk-benefit ratio of prescribing statins to elderly patients with comorbidities [37]. In Italy in particular, factors such as disability, cognitive impairment, lack of a caregiver, and a low educational level increase the probability of being untreated [35-37]. 
This study has some limitations. All patients enrolled were from the population admitted to the home care rehabilitative treatment program from a limited geographic area, which could limit the generalizability of the results obtained. Moreover, characteristics of the patients' household settings were not investigated. Finally, the results of the study need to be confirmed in a larger number of patients across a wider spectrum of clinical and sociodemographic conditions.

Our study shows that elderly patients surviving a firsttime stroke exhibit better functional recovery if they have higher TC levels. Further studies are needed to investigate and confirm this finding, and to elucidate the mechanisms underlying its positive effects.

\section{References}

[1] Guccione AA, Felson DT, Anderson JJ, et al. The effects of specific medical conditions on the functional limitations of elders in the Framingham study. Am J Public Health. 1994;84:351-358.

[2] Lefkovits J, Davis SM, Rossiter SC, et al. Acute stroke outcome: effects of stroke type and risk factor. Aust N Z J Med. 1992;22:30-35.

[3] Pettersen R, Dahl T, Wyller TB. Prediction of long-term functional outcome after stroke rehabilitation. Clin Rehabil. 2002;16:149-159.

[4] Francescutti C, Mariotti S, Simon G, D’Errigo P, Di Bidino R. The impact of stroke in Italy: first step for a National Burden of Disease Study. Disabil Rehabil. 2005;27(5):229-240.

[5] Franceschini M, Paolucci S, Perrero L, Polverelli M, Zampolini M, Giustini A. Stroke rehabilitation care in Italy. Am J Phys Med Rehabil. 2009;88:679-685.

[6] Landau WM. Is cholesterol a risk factor for stroke? Arch Neurol. 1999;56:1521-1524.

[7] Tirschwell DL, Smith NL, Heckbert SR, et al. Association of cholesterol with stroke risk varies in stroke subtypes and patient subgroups. Neurology. 2004;63:1868-1875.

[8] Shepherd J, Blauw GJ, Murphy MB, et al. Prospective study of pravastatin in the elderly at risk. Pravastatin in elderly individuals at risk of vascular disease (PROSPER): a randomised controlled trial. Lancet. 2002;360:1623-1630.

[9] Amarenco P, Bogousslavsky J, Callahan A, et al; Stroke prevention by aggressive reduction in cholesterol levels (SPARCL) Investigators. High-dose atorvastatin after stroke or transient ischemic attack. $N$ Engl J Med. 2006;355:549-559.

[10] Manktelow BN, Potter JF. Interventions in the management of serum lipids for preventing stroke recurrence. Cochrane Database of Systematic Reviews 2009, Issue 3. Art. No.: CD002091.

[11] Linee-guida del Ministro della Sanità per le attivita di riabilitazione (in GU 30 maggio 1998, n. 124)Linee di indirizzo per la definizione del percorso assistenziale ai pazienti con ictus cerebrale. Conferenza Stato-Regioni-Repertorio Atti n. 2195 del 3 febbraio 2005, Gazzetta Ufficiale n. 50.

[12] Pedersen PM, Jorgensen HS, Nakayama H, Raaschou HO, Olsen TS. Comprehensive assessment of activities of daily living in stroke: the Copenhagen Stroke Study. Arch Phys Med Rehabil. 1997;78: 161-165.

[13] Wade DT, Collen FM, Robb GF, Warlow CP. Physiotherapy intervention late after stroke and mobility. BMJ. 1992;304:609-613.

[14] Kalra L. The influence of stroke unit rehabilitation on functional recovery from stroke. Stroke. 1994;25:821-825.

[15] Mahoney FI, Barthel DW. Functional evaluation: the Barthel Index. Md State Med J. 1965;14:61-65.
[16] Green J, Forster A, Young J. A test-retest reliability study of the Barthel Index, the Rivermead Mobility Index, the Nottingham Extended Activities of Daily Living Scale and the Frenchay Activities Index in stroke patients. Disabil Rehabil. 2001;23:670-676.

[17] WHO task force. Stroke, 1989: recommendations on stroke prevention, diagnosis, and therapy. Stroke 1989;20:1407-1431.

[18] Bamford JM, Sandercock PAG, Dennis MS, Burn J, Warlow CP. Classification and natural history of clinically identifiable subtypes of cerebral infarction. Lancet. 1991;337:1521-1526.

[19] Dyker AG, Weir CJ, Lees KR. Influence of cholesterol on survival after stroke: retrospective study. BMJ. 1997;314:1584-1588.

[20] Vauthey C, de Freitas GR, van Melle G, Devuyst G, Bogousslavsky J. Better outcome after stroke with higher serum cholesterol levels. Neurology. 2000;54:1944-1949.

[21] Zuliani G, Cherubini A, Atti AR, et al. Low cholesterol levels are associated with short-term mortality in older patients with ischemic stroke. J Gerontol A Biol Sci Med Sci. 2004;59:293-297.

[22] Olsen TS, Christensen RH, Kammersgaard LP, Andersen KK. Higher total serum cholesterol levels are associated with less severe strokes and lower all-cause mortality: ten-year follow-up of ischemic strokes in the Copenhagen Stroke Study. Stroke. 2007;38:2646-2651.

[23] Li W, Liu M, Wu B, et al. Serum lipid levels and 3-month prognosis in Chinese patients with acute stroke. Adv Ther. 2008;25:329-341.

[24] Pan SL, Lien IN, Chen TH. Is higher serum total cholesterol level associated with better long-term functional outcomes after noncardioembolic ischemic stroke? Arch Phys Med Rehabil. 2010;91: 913-918.

[25] Beloosesky Y, Streifler JY, Burstin A, Grinblat J. The importance of brain infarct size and location in predicting outcome after stroke. Age Ageing. 1995;24:515-518.

[26] Johnston KC, Connors AF, Wagner DP, Haley EC. Predicting outcome in ischemic stroke: external validation of predictive risk models. Stroke. 2003;34:200-202.

[27] Simonsen U, Ehrnrooth E, Gerdes LU, et al. Functional properties in vitro of systemic small arteries from rabbits fed a cholesterolrich diet for 12 weeks. Clin Sci. 1991;80:119-129.

[28] Joseph JA, Villalobos-Molinas R, Denisova NA, Erat S, Strain J. Cholesterol: a two-edged sword in brain aging. Free Radic Biol Med. 1997;22:455-462.

[29] Chui DH, Marotta F, Rao ML, et al. Cholesterol rich LDL perfused at physiological LDL concentration induces platelet aggregation and PAF-acetylhydrolase activation. Biomed Pharmacother. 1991;45: $37-42$.

[30] Eryurek FG, Surmen E, Oner P, Altug T, Oz H. Gamma-glutamyltranspeptidase and acetylcholinesterase activities in brain capillaries of cholesterol-fed rabbits. Res Commun Chem Pathol Pharmacol. 1990;69:245-248.

[31] Dávalos A, Ricart W, Gonzalez-Huix F, et al. Effect of malnutrition after acute stroke on clinical outcome. Stroke. 1996;27:1028-1032.

[32] Ding EL, Mozaffarian D. Optimal dietary habits for the prevention of stroke. Semin Neurol. 2006;26:11-23.

[33] Fung TT, Rexrode KM, Mantzoros CS, Manson JE, Willett WC, $\mathrm{Hu}$ FB. Mediterranean diet and incidence of and mortality from coronary heart disease and stroke in women. Circulation. 2009;119: 1093-1100.

[34] Maraldi C, Lattanzio F, Onder G, et al. Variability in the prescription of cardiovascular medications in older patients: correlates and potential explanations. Drugs Aging. 2009;26(Suppl. 1):41-51.

[35] De Wilde S, Cook DG, Carey IM, Hilton SR, Whincup PH. Underuse of statins among older people. Lancet. 2003;362:746-747.

[36] Landi F, Cesari M, Onder G, et al; SILVERNET-HC Study Group. Antithrombotic drugs in secondary stroke prevention among a community dwelling older population. J Neurol Neurosurg Psychiatry. 2003;74:1100-1104. 